\title{
Búsqueda de información científica para la mejora de contenidos en protocolos de investigación en asignaturas de apoyo a la titulación en la ingeniería
}

\section{Search of scientific information for the improvement of contents in research protocols in subjects to support the engineering degree.}

Mejora de contenidos de protocolos de investigación.

María Concepción Villatoro $\mathrm{Cruz}^{(1)}$

Marina Cisneros Guerra ${ }^{(2)}$

Ingrid Monciel Lugo Martínez ${ }^{(3)}$

(1) Tecnológico Nacional de México-Instituto Tecnológico de Minatitlán. Veracruz. México. email: maria.vc@minatitlan.tecnm.mx

(2) Tecnológico Nacional de México-Instituto Tecnológico de Minatitlán. Veracruz. México. email: marina.cg@minatitlan.tecnm.mx

(3) Tecnológico Nacional de México-Instituto Tecnológico de Minatitlán. Veracruz. México. email: monciel.lugo22@gmail.com

Contacto: maria.vc@minatitlan.tecnm.mx

\section{Recibido: 30-6-2020 Aprobado: 10-9-2020}

\section{Resumen}

La formación profesional de un Ingeniero debe ser integral y cumplir con competencias que aportan en la construcción de su conocimiento, la competencia de investigación es el eje medular de este trabajo y representa el área de mejora a atender; los alumnos presentan deficiencias en la búsqueda de información científica para la generación de protocolos de investigación. Esta investigación tiene como objetivo, mejorar las estrategias de búsqueda y gestión de la información para la elaboración de protocolos 
de investigación mediante recursos tecnológicos y motores de búsqueda para obtener productos finales de calidad científica. Se aplican dos encuestas, de diagnóstico y de mejora, ésta última se aplica en dos momentos: antes de brindar soporte de contenido en la búsqueda de información y después de haberlo hecho, demostrando que se mejora la calidad de los productos obtenidos en las asignaturas de la línea de investigación. Se aplica durante 3 semestres escolares (tiempo1, tiempo2 y tiempo 3) a un total de muestra acumulado de 230 alumnos. La investigación es cuantitativa con diseño no experimental longitudinal de tendencia con un alcance descriptivo. Los resultados diagnósticos indican que Google es el recurso más utilizado por los alumnos para realizar sus investigaciones. Los resultados muestran una mejora en la calidad de los contenidos en protocolos de investigación al brindarles materiales exclusivos de ésta temática durante el curso. Se concluye que los alumnos necesitan una guía docente o de contenido para hacer búsquedas efectivas de información en espacios formales y reconocidos para el gremio científico.

Palabras claves: investigación, competencia, búsqueda de información, mejora.

\begin{abstract}
The professional training of an Engineer must be comprehensive and comply with competencies that contribute to the construction of their knowledge, the research competence is the core of this work and represents the area of improvement to be addressed; the students present deficiencies in the search for scientific information for the generation of research protocols. This research aims to improve search strategies and information management for the development of research protocols using technological resources and search engines to obtain final products of scientific quality. Two surveys are applied, diagnostic and improvement, the latter is applied in two moments: before providing content support in the search for information and after having done so, showing that the quality of the products obtained in the subjects of the line of research. It is applied during 3 school semesters (time1, time2 and time 3) to a total accumulated sample of 230 students. The research is quantitative with a non-experimental longitudinal trend design with a descriptive scope. The diagnostic results indicate that Google is the resource most used by students to carry out their research. The results show an improvement in the quality of the contents in research protocols by providing exclusive materials on this subject during the
\end{abstract}


course. It is concluded that students need a teaching or content guide to effectively search for information in formal and recognized spaces for the scientific union.

Keywords: research, competition, search for information,improvement.

\section{Instroducción}

En México como en el mundo entero el conocimiento se produce de manera continua y se renueva día a día, las Instituciones Educativas (IE) tienen la tarea de llevarlo a quienes se forman en sus magnas aulas presenciales o virtuales, así la formación profesional en los Institutos Tecnológicos pertenecientes al Tecnológico Nacional de México (TNM) siempre comprometida con la sociedad, fundamenta esta constante labor y la enmarca en el Modelo Educativo para el Siglo XXI basada en tres dimensiones: filosófica, académica y organizacional; DGEST 2012 refiere que el ámbito educativo es "donde la frontera de la investigación, del desarrollo científico, tecnológico y de la innovación se amplía continuamente" (p.13), tal documento dedica especialmente un rubro a la formación del capital humano para la investigación, la concepción del aprendizaje y la práctica educativa.

Los programas de estudio de ingeniería del TNM están basados en el modelo por competencias genéricas definidas por DGEST 2012 como un conjunto de habilidades, conocimientos, destrezas y actitudes que todo profesionista debe desarrollar y fortalecer durante su formación profesional; en este contexto, las asignaturas básicas relacionadas con la investigación: Fundamentos de Investigación, Taller de Investigación 1 y Taller de Investigación II coinciden en sus programas de estudio en al menos 3 competencias genéricas: (1) capacidad de investigación, (2) habilidades para buscar, procesar y analizar información procedentes de diversas fuentes, del cual Zachman (2015) menciona que "la gestión de información deviene en indicador de excelencia y el éxito en el contexto laboral y la investigación, en tanto cada vez más la sociedad encarga a la sociedad su dominio"(p.53), y (3) habilidad en el uso de Tecnologías de Información y Comunicación (TIC) que tienen la ventaja de tener entornos interactivos, grandes canales de comunicación y acceso flexible a la información, características de andamiaje en este trabajo con el objetivo de fortalecerlas, siendo de gran relevancia conocer las habilidades de investigación que los alumnos poseen para buscar información científica digital que soporte los productos de investigación que los programas de estudio requieren, a fin de enriquecer los contenidos textuales de 
los futuros ingenieros para estar a la altura de las exigencias sociales y laborales, dando solución a problemáticas reales fundamentadas en soportes textuales de protocolos de investigación transformándose en proyectos reales y factibles de realizarse.

Se vive en una realidad donde las TIC permiten el acceso a distintos espacios de información o fuentes de conocimiento con diferentes características que aporten valor al contenido de un protocolo de investigación, sin embargo, el alumno puede perder el objetivo en la búsqueda de la información propiciando un conocimiento disperso y erróneo que no sea significativo y asimilado por el estudiante, por ello es importante guiarles y darles a conocer metodologías de búsqueda de información científica, Kriscautzky \& Ferreiro (2014). Marrero coincide en que la información hoy es abundante, dispersa, con un crecimiento exponencial, existe una sobreabundancia y variable de calidad de la información, conceptualizándolo como “infoxicación”, (2015, p.11).

Respecto a la Búsqueda de Información RUA (2013) lo comparte como un proceso de carácter global en donde intervienen factores que se relacionan entre si y depende del tipo de información que se busca se determina el tipo de fuente de información que se consulta, sin embargo, con la inclusión de las TIC existe infinidad de información. ¿Cómo poder saber cuál información es fidedigna, fiable y de calidad?, una de las acciones es acudir a procesos de selección previos como catálogos o bases de datos. Por ello define al proceso de búsqueda de información bibliográfica como "el procedimiento que nos permite localizar, en una serie de fuentes de información distintas, un conjunto de documentos y de información necesarios para resolver cualquier problema de investigación o información que se nos plantee" (p.2).

La Universidad Nacional de Chaco Austral en Argentina, específicamente Zachman, en el 2015 realizó un trabajo respecto a las habilidades de gestión de la información y redacción científica de los estudiantes de posgrado quienes aprendieron a imitar el estilo de redacción y el método búsqueda de información de sus profesores, realizando tanto buenas prácticas de investigación de los docentes como prácticas derivadas de deficientes metodologías de búsqueda de información; con base en ese contexto se implementó un curso que estandariza la autopreparación y trabajo colaborativo de los estudiantes, con la finalidad de capacitarlos en el uso efectivo de Repositorios de Objetos de Aprendizaje (OA), motores de búsqueda de información, meta buscadores, gestores bibliográficos, directorios o índices 
para capacitarlos en la correcta redacción de producción científicos y las correspondientes publicaciónes en revistas de prestigio internacional logrando alcanzar las competencias de redacción y búsqueda de información en internet. En otro ámbito de aplicación que incluye al indicador de mejora: “deficiencia en la búsqueda de información científica en la web para productos científicos”, en el 2015 Donoso realiza un trabajo de investigación en la Universidad de Guayaquil en la carrera de comunicación social y presenta una propuesta de capacitación a los alumnos para la mejora en la redacción a fin que el alumno adquiera un conocimiento de optimización de motores de búsqueda en internet, entendiendo sus beneficios, factores claves y técnicas que el alumno ha de aplicar . Antúnez \& Veytia (2020) identifican las competencias de investigación en 10 países latinoamericanos y España mediante herramientas tecnológicas para la gestión de información científica y destacan que la accesibilidad a las TIC por parte de los docentes ha incrementado, indican la importancia de adquirir competencias investigativas en la sociedad del siglo XXI, destacando el aprendizaje autónomo. Describen como principal conclusión que la temática de investigación constituye una de las funciones prioritarias para los actores de la educación.

En el 2012 la Facultad de Educación de la Universidad de Barcelona realizó un estudio acerca del aporte de las asignaturas de investigación para el reforzamiento de trabajos finales de grado, en la perspectiva del estudiante, basándose en el modelo del Espacio Europeo de Educación Superior (EEES) que destaca la importancia de desarrollar en los estudiantes las competencias de investigación, actualmente llamadas competencias investigativas que se consideran como el eje orientados de una mentalidad científica. Uno de sus resultados está enfocado a la necesidad de búsqueda efectiva de bibliografía y fuentes de información de reconocimiento mundial, donde el 66\% de la muestra requiere ayuda para este indicador. Quirós \& Valls (2017).

Un enfoque teórico coincidente respecto a las competencias de investigación es el que presenta Vivas, Chacón y Peña (2015), a través de la perspectiva cognitivista congruente con el entorno de investigación del trabajo que se presente en este artículo, y que influenciada por las ideas de Dewey (1938) sobre el aprendizaje experiencial, comenta lo siguiente:

Destaca el lugar de los aprendices como pensadores activos y constructores de significados y busca desarrollar habilidades instrumentales para acceder, evaluar y usar información con sentido para los usua- 
rios. En este escenario se ubican, con gran trascendencia, muchas de las experiencias actuales de las Ciencias de la Información y la Documentación las que, a través de programas de formación, hacen la apuesta de contribuir con el desarrollo de habilidades informativas para apoyar el aprendizaje y la adquisición de conocimientos científicos y tecnológicos y así favorecer el desarrollo académico y personal (p.44).

Es así como se llega a la descripción puntual del quehacer de este trabajo cuyo eje de acción se origina de la observaron a los alumnos respecto a sus competencias investigativas en las asignaturas de la cadena de investigación, el diagnóstico obtenido por entrevista estructurada a 12 docentes que imparten estas asignaturas básicas ( 3 asignaturas) en las 7 carreras de ingeniería, se presenta en la siguiente conclusión integral.

Los alumnos presentan deficiencias en aterrizar contextualmente las ideas planteadas que dan origen a los productos escritos de investigación; la transformación textual de la idea al texto científico casi siempre no es expresada de forma clara y objetiva; en mediana medida se detectan errores de redacción y ortografía; la escritura se realiza en $4^{\text {a }}$ persona; dificultad para escribir en el estándar de citación APA y finalmente baja calidad en la información contenida en sus productos finales con escaso número de referencias bibliográficas utilizadas en la construcción del producto científico, en éste último indicador seleccionado se detecta el problema para esta investigación y es en donde se centra el trabajo realizado desde enero 2019 hasta junio del 2020.

Conforme a Walpole, Myers y Myers (2012), sean $\mu 1$ y $\mu 2$ los valores promedios para la medición de la mejora y en las que se basa la siguiente hipótesis: la calidad en los contenidos de productos científicos mejora al proporcionar a los alumnos estrategias tecnológicas para la búsqueda de información científica.

El presente artículo tiene por objetivo mejorar las estrategias de búsqueda y gestión de la información para la elaboración de contenidos de protocolos de investigación mediante recursos tecnológicos y motores de búsqueda para obtener productos finales de calidad científica.

\section{Materiales y métodos}


El enfoque es cuantitativo con diseño no experimental longitudinal de tendencia con un alcance descriptivo, ya que el proyecto se aplicó en 3 semestres consecutivos con diferentes muestras distintas de alumnos cada semestre, con la misma población, definiendo los bloques tiempo 1 :recolección de datos de una población (70 alumnos en el semestre enero-junio 2019, 2 grupos), tiempo 2 :recolección de datos de una población (85 alumnos en el semestre agosto-diciembre 2019, 3 grupos) y tiempo 3 :recolección de datos de una población (75 alumnos en el semestre enero-junio 2020, 2 grupos). Cabe aclarar que este trabajo representa la primera etapa de ejecución aplicándose a 3 carreras sólo en las asignaturas básicas de investigación (230 alumnos) y en una segunda etapa se aplicará a las carreras restantes que se ofertan en nuestro instituto.

Como primera acción para cada grupo se reconoce el nivel de competencia de investigación al inicio de cada semestre aplicando una encuesta diagnóstica en Google Forms acerca del contexto en el uso de tecnologías para la búsqueda de información y detectando cuales son los espacios que los alumnos utilizan para el desarrollo de sus protocolos, a continuación se inicia con las actividades de enseñanza y aprendizaje que cada docente planea en las instrumentaciones didácticas de cada asignatura; durante el primer tema de las asignaturas se solicita que realicen la primera etapa de su protocolo generando título, introducción, justificación, objetivos, marco teórico y metodología, con las instrucciones descritas en la actividad de aprendizaje realizándolas sólo con las competencias hasta ahora aprendidas en su formación, esta actividad la evalúa el docente con rúbrica de 8 indicadores (variables), de los cuales 3 se relacionan con la búsqueda de información: (1) elección de fuentes relevantes, (2) inserción de referencias recientes y (3) calidad en la estructura de la revisión literaria. Se evalúa la primera versión de la etapa (versión 1 del texto científico), se obtienen resultados de la evaluación, se pone atención a los 3 indicadores descritos anteriormente. Aquí sucede el momento $l(\mu 1)$ de la aplicación de la encuesta de mejora en Google Forms a los grupos evaluados, validada por método de agregados individuales, Corral (2009) y aplicando el método de estabilidad (test-reset). Se obtienen los primeros resultados. A continuación se dedica una semana completa al interior de cada grupo a realizar un taller de competencias de búsqueda de información científica diseñado y desarrollado en enero del 2019 por 2 docentes y un residente del área de sistemas, se agregan dos semanas se asesoría y seguimiento para obtener el producto textual del taller impartido a los alumnos y actualizando de ser necesario la versión1 de la primera etapa, el alumno aplica lo aprendido a su primer producto, transformándolo en una etapa 2 (ver- 
sión 2 del texto científico). El docente evalúa nuevamente con la misma rúbrica y obtiene resultados. Aquí sucede el momento $2(\mu$ 2) de la aplicación de la encuesta de mejora al grupo evaluado. Se utiliza PSPPIRE, para en análisis de datos obtenidos. Para comparar los resultados obtenidos de los estudiantes antes y después de la actividad de aprendizaje-taller se aplica la prueba estadística T de Student para grupos relacionados emparejados.

\section{Resultados}

Uno de los bloques de la encuesta diagnóstica es llamada Búsqueda de información y está integrada por los siguientes indicadores: 1. Motores de búsqueda en Google: motores de búsqueda google como primera opción de búsqueda de información, 2. Acceso a REA: ha ingresado al menos a 2 los repositorios educativos abiertos y/o bases de datos de información científica como CONACYT, UNAM, CONRICYT, Dialnet, Redalyc, Scielo, SCOPUS, EBSCO, Bit, proyecto Gutenberg u otros, 3. Información en otros idiomas: busca información científica en otros idiomas distintos al español, 4. Información reciente; cuando busca información en la web, se interesa por que sea información reciente (no más de 5 años) y 5. Número de fuentes utilizadas: utiliza al menos 5 fuentes de información como mínimo en sus trabajos académicos. Los tiempos registrados son: tiempo 1 comprende el semestre enero-junio 2019 con 70 alumnos, tiempo 2 comprende el semestre agosto-diciembre 2019 con 85 alumnos y tiempo 3 comprende el semestre Enero-junio 2020 con 75 alumnos. Los resultados se presentan en la gráfica 1.

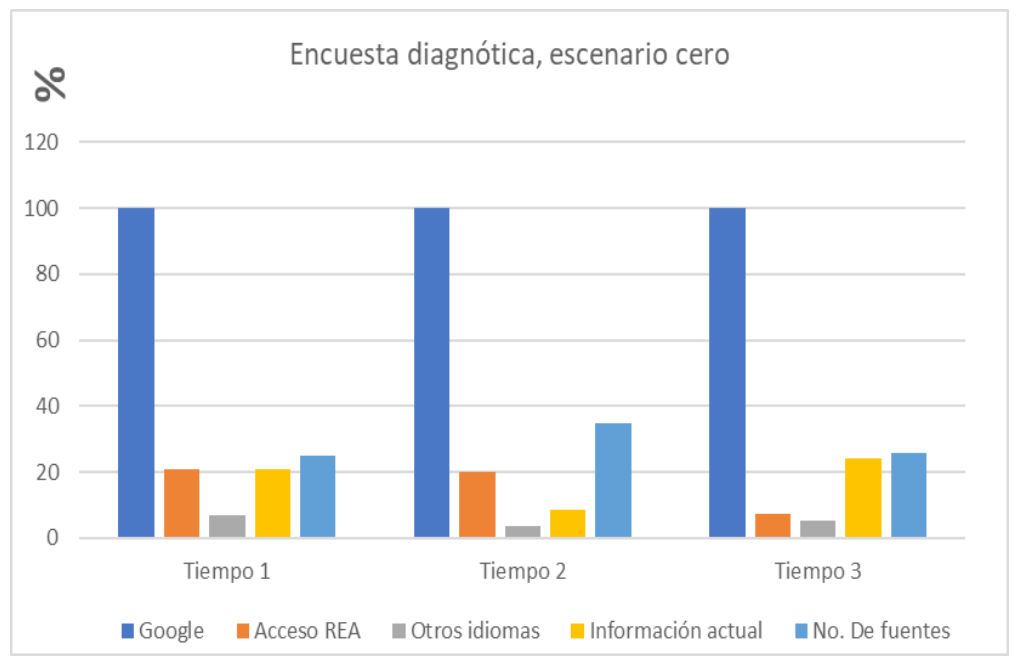

Figura 1. Resultados del bloque "Búsqueda de información”, encuesta diagnóstica. 
La gráfica 1, muestra en 5 ítems el área de oportunidad en el fortalecimiento de las competencias investigativas con tecnología en espacios digitales, los niveles obtenidos están por debajo de la media por lo que se verifica la necesidad de reforzar ésta área para extender el contexto de espacios confiables e idóneos para la búsqueda de información científica.

La segunda encuesta aplicada y que proporciona los datos más relevantes al respecto de la mejora que plasma la hipótesis es la aplicación de la encuesta de mejora donde se muestran los resultados de los momentos 1 (versión 1 del protocolo de investigación antes del taller formativo) y 2 (versión 2 del protocolo de investigación, después del taller formativo) para un nivel de confianza de 95\% con riesgo de 5\% ( $\alpha=0.05), t>2.1788$. Según los resultados analizados en PSPPIRE, se comprueba la hipótesis si $\mu 2$ $>\mu 1$, demostrando que existe una mejora en la calidad de contenidos de los productos científicos por lo que se comprueba la hipótesis establecida. En la tabla 1 se muestran los resultados en función de los 3 indicadores principales de este proyecto: elección de fuentes relevantes, inserción de referencias recientes y calidad en la estructura de la revisión literaria.

\begin{tabular}{|l|l|l|l|l|}
\hline Tiempo de aplicación & $\begin{array}{l}\text { No. de } \\
\text { muestra }\end{array}$ & $\mu 2$ & $\begin{array}{l}\mu 1(2.1788) \& \\
\mu 2\end{array}$ & $\begin{array}{l}\text { iSe evidencia } \\
\text { mejora? }\end{array}$ \\
\hline $\begin{array}{l}\text { Tiempo 1: Semestre Enero- } \\
\text { Junio 2019 }\end{array}$ & 70 & 17.33 & $\mu 2>\mu 1$ & $\mathrm{Si}$ \\
\hline $\begin{array}{l}\text { Tiempo 2: Semestre Agosto- } \\
\text { Diciembre 2019 }\end{array}$ & 85 & 18.01 & $\mu 2>\mu 1$ & $\mathrm{Si}$ \\
\hline $\begin{array}{l}\text { Tiempo 3: Semestre Enero- } \\
\text { Junio 2020 }\end{array}$ & 75 & 17.88 & $\mu 2>\mu 1$ & $\mathrm{Si}$ \\
\hline
\end{tabular}

Tabla 1. Resultados del análisis de datos comprobatorias de la mejora.

Se describe en la tabla 2, de manera más puntual y descriptiva los porcentajes de la mejora en función de la evaluación que realiza el docente a los protocolos de investigación.

Según la tabla 2 se observa que ha disminuido el porcentaje de reprobación (nivel 1) en un promedio del 16\%; respecto al nivel 2 se observa una mejora del $11 \%$, y en el rubro del nivel 3 la mejora promedio es de 6\%. Se destaca que en el nivel 2 la mejora es aceptable considerando que para la mayoría de los alumnos es la primera vez que conocen un entorno amplio de fuentes de información y que con la 
práctica pueden mejorar hasta la excelencia en la redacción de los protocolos de investigación, así como en el nivel 3.

\begin{tabular}{|l|l|l|l|l|l|l|l|l|l|}
\hline \multicolumn{2}{|l|}{ Tiempo 1 } & \multicolumn{2}{l|}{ Tiempo 2 } & \multicolumn{2}{l|}{ Tiempo 3 } \\
\hline $\begin{array}{l}\text { Niveles de eva- } \\
\text { luación del proto- } \\
\text { colo }\end{array}$ & $\mu 1$ & $\mu 2$ & $\begin{array}{l}\text { Mejo- } \\
\text { ra }\end{array}$ & $\mu 1$ & $\mu 2$ & $\begin{array}{l}\text { Mejo- } \\
\text { ra }\end{array}$ & $\mu 1$ & $\mu 2$ & $\begin{array}{l}\text { Mejo- } \\
\text { ra }\end{array}$ \\
\hline $\begin{array}{l}\text { Nivel 1: } \\
\text { Calificación me- } \\
\text { nor de } 70\end{array}$ & $24 \%$ & $9 \%$ & $15 \%$ & $22 \%$ & $5 \%$ & $17 \%$ & $19 \%$ & $4 \%$ & $15 \%$ \\
\hline $\begin{array}{l}\text { Nivel 2: } \\
\text { Calificación entre } \\
71 \text { y 85 }\end{array}$ & $60 \%$ & $70 \%$ & $10 \%$ & $58 \%$ & $69 \%$ & $11 \%$ & $67 \%$ & $78 \%$ & $11 \%$ \\
\hline $\begin{array}{l}\text { Nivel 3: } \\
\text { Calificación entre } \\
86 \text { y 100 }\end{array}$ & $16 \%$ & $21 \%$ & $5 \%$ & $20 \%$ & $26 \%$ & $6 \%$ & $11 \%$ & $18 \%$ & $7 \%$ \\
\hline
\end{tabular}

Tabla 2. Resultados de la evaluación del protocolo realizada por el docente expresado en porcentajes de mejora.

\section{Discusión}

En la interpretación de los resultados obtenidos de denota como primer hallazgo que los alumnos no tienen una preparación adecuada en el nivel medio superior en el ámbito de las competencias de investigación, dando como resultado bajos niveles de aprendizaje y en consecuencias se reflejan en los niveles de reprobación en las asignaturas del contexto investigativo. También resultó importante la existencia de distractores para la búsqueda de información científica y tecnológica y en este tenor Vivas, Chacón \& Peña (2015) destacan en los resultados de su trabajo la importancia de los recursos que se utilizan, la participación de los docentes, para mejorar la comprensión de grandes cantidades de información, la introducción de actividades lúdicas para el desarrollo de competencias de investigación tiene un valor importante para captar la atención y motivación de los estudiantes en mejorar sus textos científicos. (p.61).

Se concuerda con la investigación de Antúnez, Pellicer, Valera, Sánchez, Ollarzábal, \& Alés (2012) que impartieron un curso virtual de redacción científica aplicado a un grupo de docentes tal que contri- 
buye a la adquisición de habilidades para redactar artículos científicos (p.11); y en efecto, este trabajo valida que con la aplicación de una estrategia formativa dedicada a la búsqueda de información científica con todos los elementos necesarios para enseñar (motores de búsqueda, REA, bases de datos, metabuscadores, repositorios institucionales, etc.), se puede mejorar la calidad de trabajos científicos en los alumnos de una institución educativa.

En esta experiencia se coincide con los hallazgos de los autores George, \& Salado (2019), revelan que los estudiantes emplean con frecuencia las tecnologías con fines comunicativos y de búsqueda de información de Internet.

\section{Conclusiones}

El tema de la investigación es medular en toda sociedad que busca solucionar problemáticas del entorno, la preparación de futuros ingenieros es fundamental para que ellos contribuyan en este valioso eje académico, las Universidades y Tecnológicos proponen diversas estrategias de mejora según su entorno, recursos y escenarios propios de cada núcleo educativo; la investigación aquí compartida toca a una parte de ese gremio ingenieril y se demuestra que se puede atender vacíos académicos con propuestas ad hoc con las herramientas que poseen los beneficiarios de la educación. Es así como el desenlace del trabajo aporta una mejora en el ámbito de la enseñanza y fortalecimiento de la competencia de investigación al incluir entre el semestre una formación dedicada a la enseñanza de búsqueda de información en escenarios digitales, el aporte va desde disminuir de manera moderada los índices de reprobación, hasta elevar los índices de calificaciones mayores de $81 \%$ en los alumnos que cursan las asignaturas de la cadena de investigación en el Instituto Tecnológico de Minatitlán. Otra conclusión importante es que los alumnos requieren de una guía docente o de contenido para realizar búsquedas de información efectiva y lograr el objetivo de desarrollar contenidos de calidad para los protocolos de investigación.

De manera particular se analiza el comportamiento del nivel 2 de calificaciones obtenidas (entre $71 \%$ y $85 \%$ ) y se detecta que el nivel de mejora es prudente, conocer los factores será motivo de otra investigación, empero se puede prospectar como factor la poca experiencia que los alumnos tienen al redactar 
y buscar información científica, ya que, para el mayor porcentaje de los alumnos, estas asignaturas representan su primera experiencia con el mundo de la investigación formal, tal cual debe ser.

\section{Bibliografía}

Antúnez, A. G., \& Veytia Bucheli, M. G. (2020). Desarrollo de competencias investigativas y uso de herramientas tecnológicas en la gestión de información. Revista Conrado, 16(72), 96-102. Recuperado de http://conrado. ucf.edu.cu/index.php/Conrado

Antúnez, G., Pellicer, Y., Valera, Y., Sánchez, W., Ollarzábal, Á., \& Alés, A. (2012). Curso virtual de redacción científica e infotecnología sobre la plataforma Moodle: resultados y experiencias. PíxelBit. Revista de Medios y Educación, (41), 173-183. Recuperado de: https://www.redalyc.org/ pdf/368/36828247013.pdf

Corral. (2009). Validez y confiabilidad de los instrumentos de investigación para la recolección de datos.

DGEST (2012). Modelo educativo para el siglo XXI. Formación y desarrollo de competencias profesionales. ISBN: 978-607-7912-20-0. Impreso en México. Recuperado de: https:// www.tecnm.mx/director-general/modelo-educativo-para-el-siglo-xxi-formacion-y-desarrollo-decompetencias-profesionales-dp2

Donoso Moreno, j. E. (2017). Análisis de la redacción periodística y uso de la tecnología digital, en los estudiantes del primer semestre de la carrera de Comunicación Social en el año 2015 (Doctoral dissertation, universidad de Guayaquil). Recuperado de: http://repositorio.ug.edu.ec/ bitstream/redug/15313/1/TESIS\%20JORGE\%20DONOSO.pdf

George, C. \& Salado, L. (2019). Competencias investigativas con el uso de las TIC en estudiantes de doctorado. Apertura (Guadalajara, Jal.), 11(1), 40-55. https://doi.org/10.32870/ap.v11n1.1387

Ramos, E. (2015) "Prueba T de Student para datos relacionados (muestras dependientes)," (en línea), consultada por Internet el 30 de junio del 2015. Recuperado de http:/www.ray-design.com.mx/ 
psicoparaest/index.php?option $=$ com_content\&view $=$ article\&id $=232:$ t-student-dr\&catid $=52$ :pruebaspara\&Itemid $=61$.

Hernández, R., Fernández, C. \& Baptista, M. (2010). Metodología de la Investigación. McGraw Hill.

Kriscautzky, M., \& Ferreiro, E. (2014). La confiabilidad de la información en Internet: criterios declarados y utilizados por jóvenes estudiantes mexicanos. Educação e Pesquisa, 40(4), 913-934.

Marrero Sera, E. (2015). Manejo de bases de datos competencias en la búsqueda y acceso a la información digital I. Recuperado de: https://repositorio.unphu.edu.do/handle/123456789/919

Quirós Domínguez, c. L., \& Valls Figuera, r. G. (2017) El aporte de las asignaturas de investigación y los aspectos a reforzar en la formación para la realización del trabajo de fin de grado de pedagogía. La percepción de los estudiantes.

R. E. Walpole, R. H. Myers y S. L. Myers. (1999) "Probabilidad y Estadística para Ingenieros, 6a ed.," Prentice-Hall Latinoamericana, México, 1999.

RUA. Biblioteca Universitaria de Alicante (2013). La búsqueda de información científica. Recuperado de https://rua.ua.es/dspace/bitstream/10045/33983/1/BUSQUEDA_informacion_DOCTORADO.pdf

Vivas, G. P. M., Chacón, J. W. B., \& Peña, H. C. (2015). Desarrollo de competencias informacionales en contextos universitarios: enfoques, modelos y estrategias de intervención. Investigación Bibliotecológica: archivonomía, bibliotecología e información, 29(65), 39-72. Recuperado de: https://www.sciencedirect.com/science/article/pii/S0187358X16000150

Walpole, R. E., Myers, R. H., Myers, S. L., \& Ye, K. (2012). Probabilidad y estadística para ingeniería y ciencias. Norma, 162, 157.

Zachman, P. P. (2015). Infotecnología en la Formación de Posgrado. In X Congreso sobre Tecnología en Educación \& Educación en Tecnología (TE \& ET) (Corrientes, 2015). Recuperado de: http:// 
Revista Sinapsis. Vol 3, Nro 18, diciembre de 2020 | ISSN 1390-9770

sedici.unlp.edu.ar/bitstream/handle/10915/48965/Documento_completo.pdf?sequence=1\&isAllo$\underline{w e d}=y$ 